\title{
Índice de autores
}

\section{A}

\begin{tabular}{ll} 
Abajo M & 84 \\
Aguilera Guirao A & 83 \\
Alende-Castro V & $85,90,98$ \\
Álvarez-Díaz H & 80,86 \\
Álvarez Fernández M & 83 \\
Álvarez Otero J & $82,85,87,91,93,97$ \\
Alves Pereira ED & $82,87,88,89,97$ \\
Anibarro García L & 89 \\
Anibarro L & $81,82,89,97$ \\
Antela López A & 80 \\
Araújo Fernández S & $82,91,97$ \\
Arca Blanco A & 87 \\
Arévalo A & $83,92,97$ \\
Argibay Filgueira AB & $80,84,86,87,89,91,92$ \\
Arias Castaño JC & 90 \\
Arroyo D & 84 \\
Asensio P & 92 \\
Atienza Pintos A & 86 \\
B & \\
Baamonde I & 91 \\
Barbagelata C & $83,92,97$ \\
Barbagelata López C & 97 \\
Baroja Basanta AL & $83,84,86,87,89,91$ \\
Barreiro Rivas A & $81,82,85,93$ \\
Bellas P & 84 \\
Bellido Guerrero D & 83,92 \\
Bermúdez Ramos M & $80,83,90,98$ \\
Betriu A & 84 \\
Bravo Blázquez I & 92 \\
Brea Aparicio R & $82,87,88,89$ \\
Brea R & 97 \\
Buño Ramilo B & 97 \\
\hline &
\end{tabular}

C

Calviño L

Canitrol López A $\quad 86$

Canitrot López A

Canneti B 81

Caro C

Castelo Corral L $\quad 88$

Castro A $\quad 81,83,87$

Castro D 84

Cerqueiro Gonzalez JM 80

Cimas I 84

Clavero E $\quad 83,87,91$

Clavero Fernández $\mathrm{E} \quad 89$

Corredoira-Sánchez J 93

Dalama López T 92

De La Calle Otero A 86

De la Fuente Aguado J $82,84,85,87,90,91,93$,

o la Fuente Iglesias M 90

De Lis JM 82

De Toro Santos M 93

Díaz Díaz JL 81,84

Domínguez Rodríguez T 81, 84, 89

Domínguez T 84

Dubois Silva A $\quad 81,82$

E

Encabo González A $\quad 81,82,87,88,89,97$

Enríquez Gómez H $\quad$ 87, 91,93

Estevez Gil M
F

Fernandez Castro $\quad 84$

Fernández Fernández FJ 84, 91

Fernández-Giráldez E 84

Fernández González A 86, 89

Fernández-Piñeiro JC 93

Fernández Regal I $\quad 82,85,93$

Ferro Aboy M 86

Freire Dapena M $\quad 84,89,92$

Freire M

Freire Pereira MT 86

Freire S 83, 92, 97

\section{G}

García Alén D 92

García-Garrota F 93

García Gromaz D $\quad 83,92$

García JC $\quad 80,82,87,89$

García N

García Poutón N $\quad 82,84,85,90$

García-Rodríguez JF $\quad 80,86$

García Román M 93

García-Villafranca A $\quad 85,90,98$

Gómez Buela I $\quad 92$

Gómez S 90

Gómez Sousa JM 86, $\quad$ 81, 92

Gondar Barcala T $\quad 82,90,91,93$

González Fernández D 86

González González L $\quad$ 82, 85, 97

González Noya A $\quad 82,85,93$

Gonzalez Quintela A $\quad 83,91$

González V 97

González Vázquez L $\quad 87,93$

González Vigo V 84

\section{I}

ribarren M $\quad 83,88$

Labajo L

83,88

Lado Lado F

Lamas Ferreiro JL

$82,85,97$

Latorre Díez A

$82,85,93$

Ljo Carballeda C $\quad 81,92$

Llinares D $\quad 83,92,97$

Llinares Mondéjar $\mathrm{P} \quad 89$

Longueira R 83, 86, 88, 90,93

López Calvo $S \quad 90,91$

López Mato P 82, 85,93

López-Reboiro ML $\quad 80,81,93$

López $S$

81

Lorenzo Castro R $\quad 84,86,91$

Lorenzo-Castro $R$

Lorenzo JR

Losada Arias E

\section{0}

M

Macía-Rodríguez C $\quad 80,81,85,90,98$

María Justo Muradas I 84

Mariño-Callejo A $\quad 80,86$

Marta Rodrígez $\quad 80$

Martínez A 87

Martínez-Braña L $\quad 90,98$

Martínez de Rituerto ST $\quad 80,83$

Martínez J $\quad 87$

Martínez Lamas L $\quad 83$

Martínez Muradás AJ 88

Martínez-Rey C $\quad 85,90,91$
Martínez Vidal A

Martín Malmierca N

$83,89,91,92$

Mateo-Mosquera L

81,84

Matilde Trigo

Meijide $\mathrm{H}$

Mella Pérez C

Mena A

Mengual-Macenlle N

Miralles C

Monreal M

Montes Santiago J

Moreno JA

Mouronte C

N

Naval Calviño MG 93

Nodar Germiñas A 83

Novoa Lamazares L $\quad 84,87$

Núñez Fernández JM 87

Nuñez M

Núñez MJ

97

o

Ocampo A

$80,81,82,87,89$

Otero Antón E

$83,88,90$

80,83

Páez-Guillán EM

Pato A

Paz Ferrín J

Pazos MC

Pedreira JD

Penas Ríos JL

Pérez Carral V

Pérez Piñeiro A

Pérez Rodriguez M

Pernas B

Piñeiro-Fernández JC

Piñeiro $P$

Piñeiro Parga P

Pita J

Porteiro J

Portela D

Portela Orjales D

Porto Pérez AB

Posada García J

Pose A

Poveda E

Puente Hernandez M

Puerta Louro $\mathrm{R}$

Puig Saez MA

Puy A

Rabuñal-Rey R

Ramos L

Rey Fariña S

Rico P

Rios-Prego M

Ríos Prego M

Rivas MD

Rivera A

Rivera Gallego A

Rodrígez M

Rodríguez A

Rodríguez Arias M

Rodríguez García S

Rodríguez Gómez A

Rodríguez Gómez AM

Rodríguez I

$85,90,98$

84

83,88

$81,83,87$

$82,85,93$

88

$81,83,87$

80,81

$83,92,97$

84,90

93

92

93

83

$81,84,91$

85

9

$81,83,87$

91

$82,87,93,97$

81
Rodríguez-Osorio I

Rodríguez S

Romay-Lema EM

Ruanova Suarez S

Rubinos-Chaos $\mathrm{P}$

$\mathbf{S}$

Saborido Froján J $\quad 84$

Sánchez Conde P $\quad$ 82, 85, 87, 93

Sánchez Vidal E $\quad 88,9$

Sanjurjo Rivo A $\quad 87$

Seonae González AB

Serrano J

Sesma Sánchez P $\quad 92,97$

Sesma-Sánchez $P \quad 80,86$

Sopeña B

Soto González A

Soto Peleteiro A

Sousa Domínguez A

Sousa-Domínguez A

Suárez Fuentetaja R

Suárez Gill R

Suárez-Gill R

Suárez $R$

Tato $\mathrm{R}$

V

Valcarce-Pardeiro $\mathrm{N}$

Valdivielso JM

Val Domínguez N

Valle Feijoo L

Valls J

Val N

Varela Vaamonde JA

Vares González M

Vares M

Vargas J

Vázquez $\mathrm{P}$

Vázquez $\mathrm{R}$

Vázquez Rodríguez $\mathrm{P}$

Vázquez-Triñanes $C$

Vázquez Vázquez B

Vega Riveiro $P$

Velo A

Velo García A

Velo-García A

Ventura-Valcárcel P

Verdeal Dacal R

Vilariño-Maneiro L

Vilas B

Vilas Pio B

Villaverde-Álvarez |

Villaverde I

$80,81,93$

91

80

83,92

$83,86,91,92$

80

81

$80,81,93$

83,87

81

80,86

84

86,87

86

91

$83,92,97$

81

90,9

97

$81,84,88,89,90,91$

$81,83,84,86,89,91,92$ 